\title{
R\&D for the PANDA Barrel DIRC
}

\author{
Maria Patsyuk* \\ GSI, Darmstadt, Germany \\ Goethe Universität, Frankfurt am Main, Germany \\ E-mail: m.patsyuk@gsi.de
}

The PANDA experiment at the new Facility for Anti-proton and Ion Research in Europe (FAIR) at GSI, Darmstadt, will study fundamental questions of hadron physics and QCD using highintensity cooled anti-proton beams with momenta between 1.5 and $15 \mathrm{GeV} / \mathrm{c}$. Efficient Particle Identification (PID) for a wide momentum range and the full solid angle is required for reconstructing the various physics channels of the PANDA program. Hadronic PID in the barrel region of the detector will be provided by a DIRC (Detector of Internally Reflected Cherenkov light) counter. The design is based on the successful BABAR DIRC with important improvements, such as focusing optics and fast photon timing. A detailed detector simulation is performed using Geant. A reconstruction algorithm was developed to quantify the performance of different design options in terms of single photon Cherenkov angle resolution and photon yield. Several geometrical improvements, including different radiator geometries and optics, were tested in particle beams at GSI and CERN. In this contribution simulation and reconstruction, the design options, and performance of the detector prototype will be discussed.

XXI International Baldin Seminar on High Energy Physics Problems, September 10-15, 2012

JINR, Dubna, Russia

${ }^{*}$ On behalf of the PANDA Cherenkov group. 


\section{Introduction}

The international accelerator Facility for Anti-proton and Ion Research in Europe (FAIR) [7] in Darmstadt is being constructed to provide high-quality anti-proton beams to many experiments. One of the main experiments designed to study the nature of the strong interactions in detail is PANDA. It will use an internal target to investigate anti-proton annihilation in the momentum range of $1.5-15 \mathrm{GeV} / \mathrm{c}$. The multi-purpose detector has almost $4 \pi$ acceptance and can be divided into two main parts: the Target Spectrometer based on the superconducting solenoid magnet surrounding the interaction point and the Forward Spectrometer, based on a dipole magnet, for small polar angle tracks. Studying the quark confinement using charmonium spectroscopy will help to understand why free quarks are not observed. The properties of the color neutral states will be investigated using the predictions of quantum chromodynamics for exotic states such as $q q q q \bar{q}$ or $q q \bar{q} \bar{q}$, gluonic excitations and multi-quark systems. One of the most puzzling question of modern physics, how the hadrons obtain their mass, will be addressed by studying meson properties in nuclear matter and restoration of chiral symmetry. The benchmark channels are, for example, the decays of mesons (and other hadrons) with hidden and open charm. Most of the interesting reactions have kaons and pions in their final state, which means that one of the important detector requirements will be an excellent hadron particle identification (PID) system for efficient separation of kaons from pions.

\section{Hadron PID for PANDA}

Hadron PID for the Target Spectrometer will be performed by two DIRC (Detection of Internally Reflected Cherenkov light) [2] detectors: the Barrel DIRC and the Disc DIRC, respectively, which are shown in Fig. 1. The Barrel DIRC [3] was inspired by the successful BABAR DIRC at SLAC, which has been playing a significant role in almost all the BABAR physics analyses and showed stable and reliable operation for more than 8 years [5]. Several core features of DIRCs are of particular importance for PANDA. The DIRC is small in radial direction both in size and in radiation length, which reduces the amount of material in front of the electromagnetic calorimeter and allows making it, as well as the solenoid magnet, more compact and affordable. The resulting hit pattern together with the reconstructed particle track contains the information about the Cherenkov angle and hence about the speed of the charged particle. Ultimately, the particle identification based on the DIRC detectors provide likelihoods for signals to originate from an electron, muon, pion, kaon or proton.

\section{The PANDA Barrel DIRC}

The principle of the DIRC counter is based on the Cherenkov effect. A charged particle with velocity $\beta=v / c>1 / n$, propagating in a medium with refractive index $n$, emits Cherenkov photons on a cone, where the opening angle is $\theta=\operatorname{acos}(1 /(\beta n(\lambda)))$. A key feature of DIRCs is that a radiator is also a light guide. The rectangular radiators of the Barrel DIRC are made of synthetic fused silica with $n \sim 1.47$ so that there are always some totally internally reflected photons for $\beta \sim 1$ particle. These are guided along the bar to the readout end, conserving the magnitude of 


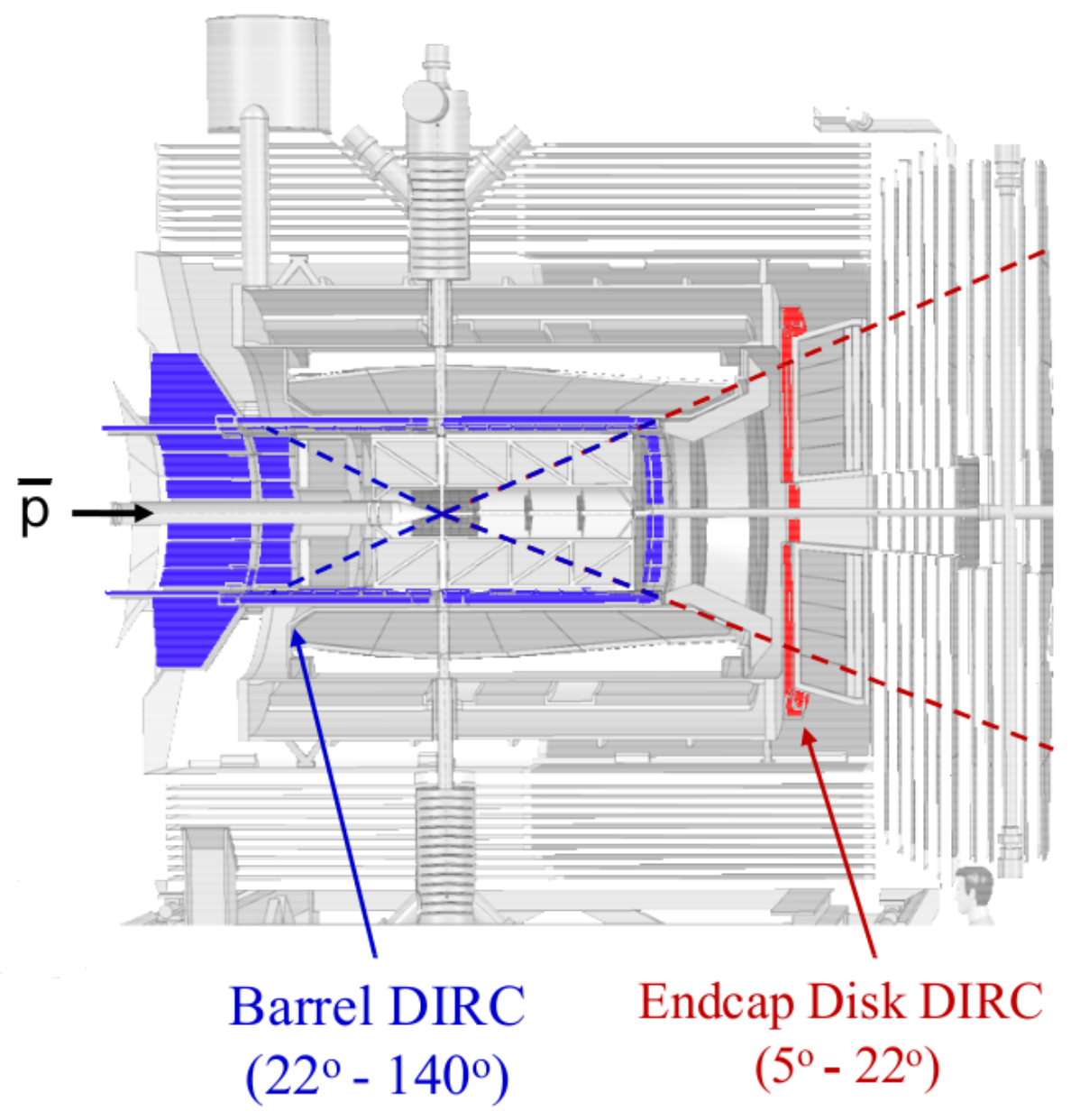

Figure 1: PANDA Cherekov counters in the Target Spectrometer.

the Cherenkov angle due to the high quality of the radiator surfaces in terms of their planarity and perpendicularity. Photons propagating towards the forward end are reflected at a mirror, and thus also enter the expansion volume through a focusing system and are imaged on a photon detector plane. The DIRC delivers information not only about the position of the detected photon on the pixelized plane, but also the hit time, which can be used for chromatic error correction. Therefore, the DIRC is intrinsically a 3-D device.

The PANDA particle identification requires at least three standard deviations separation between pions and kaons in the momentum range from $0.5 \mathrm{GeV} / \mathrm{c}$ up to $3.5 \mathrm{GeV} / \mathrm{c}$ (see [3]). Although the baseline design of the PANDA Barrel DIRC is based on the BABAR counter, some important improvements are to be implemented: a more compact expansion volume together with a focusing system, and fast photon timing.

The detailed description of the current detector design can be found in [3]. The general layout for the PANDA Barrel DIRC, shown in Fig. 2, consists of 16 bar boxes with 5 radiator bars each placed at a radius of $47.6 \mathrm{~cm}$ from the beam line. Each bar has the dimensions $17 \mathrm{~mm} \times 33 \mathrm{~mm} \times 2500 \mathrm{~mm}$. An air gap separates the lens from the expansion volume, which has a depth of $30 \mathrm{~cm}$ and is filled with oil to match the refractive index of synthetic fused silica. An array 


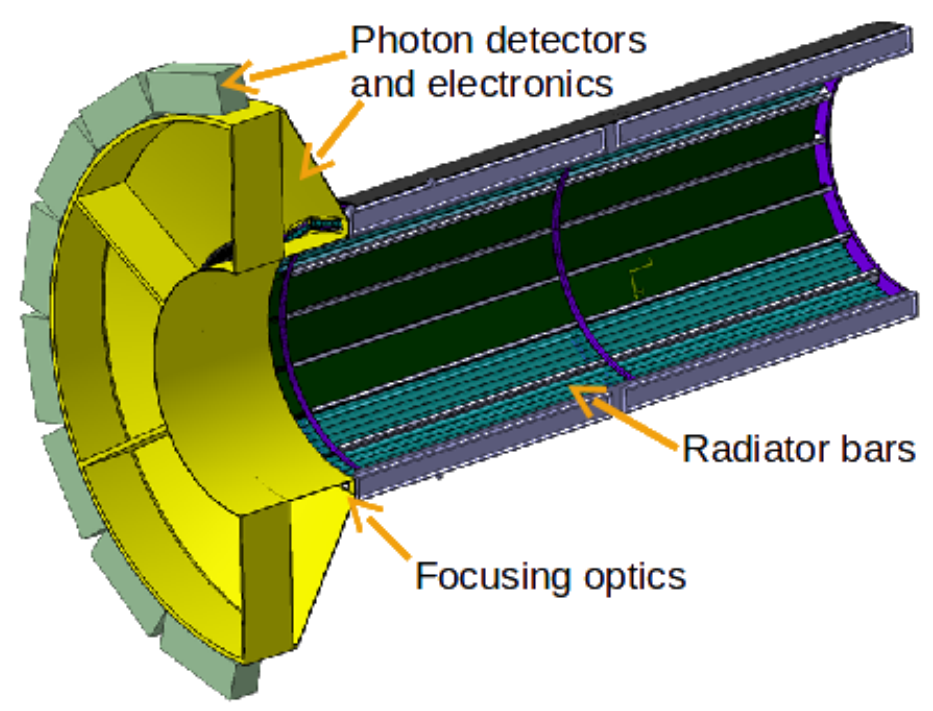

Figure 2: CAD-drawing of the Barrel DIRC counter including the support structure.

of Microchannel-PMTs (MCP-PMTs) with $10000-15000$ channels in total covers the imaging plane of the expansion volume.

Many parameters need to be optimized, and a number of design options are being studied, to achieve the design resolution and reduce the detector cost. There are two general ways to test the performance of a given design: simulation and test experiments.

\section{Simulation of the PANDA Barrel DIRC}

The detailed detector simulation is implemented using Geant [4]. Fig. 3 shows the signature

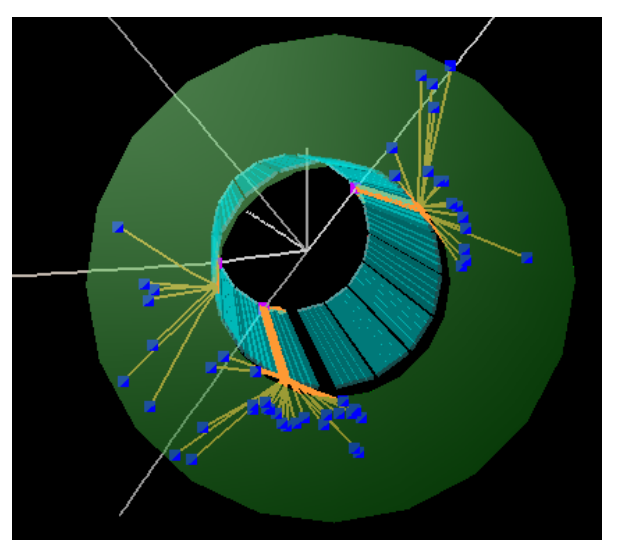

Figure 3: The PANDA Barrel DIRC in simulation: charged muons (gray) hit the radiator bars and produce Cherenkov photons (orange), that propagate along the bar and hit the photon detector (hit pixels are shown in blue). of three muon tracks in the PANDA Barrel DIRC. In addition to the baseline design a number of different design options are implemented. Some critical parameters to be studied with the simulation are: width and thickness of the radiator bars, type of the focusing system and shape of the expansion volume.

The Cherenkov angle resolution can be written as the following:

$$
\sigma_{\text {track }}=\sigma_{\text {corr }} \oplus \frac{\sigma_{\theta_{C}}}{\sqrt{N_{\text {photons }}}},
$$

where $\sigma_{\text {track }}$ is the Cherenkov angle resolution per track, $\sigma_{\text {corr }}$ includes the correlated errors coming from the tracking and multiple scattering, $\sigma_{\theta_{C}}$ is the single photon Cherenkov angle resolution, and $N_{\text {photons }}$ is the photon yield. 
Taking into account the smallest difference in the Cherenkov angle between pions and kaons in the momentum range from 0.5 to $3.5 \mathrm{GeV} / \mathrm{c}$, which is about $9 \mathrm{mrad}$, the corresponding Cherenkov angle resolution per track should be less than $3 \mathrm{mrad}$. The contribution from the PANDA tracking system is estimated to be about $1 \mathrm{mrad}$. A proven BABAR-type reconstruction was implemented to quantitatively evaluate the second term in the Eq. 4.1. It utilizes the unique DIRC property, that the positions of the hit pixel and the center of the readout bar end can be used to approximate the initial direction of the detected photon up to a number of discrete ambiguities. This photon direction vector is determined in simulation by placing a photon source at the end of the bar and illuminating the entire detector plane. The average direction vector is determined for each detector pixel and saved in a look-up table. During the reconstruction stage this vector is combined with the particle direction, measured by the tracking system, to obtain the Cherenkov angle per photon. The width of the distribution for the single photon Cherenkov angle represents the single photon Cherenkov angle resolution (SPR).

This method was applied to a simplified detector design, where no focusing is used and the bars are directly attached to the expansion volume. To obtain the single photon Cherenkov angle resolution for different track directions, relativistic muons were generated on a grid in $\left(\phi_{\text {track }}, \theta_{\text {track }}\right)$ space covering one bar box. The SPR was reconstructed for each set of track angles on this grid (see the upper plot in Fig. 4), and the average number of photons per track was calculated (the middle plot in Fig. 4). Several effects contribute to the observed SPR non-uniformity: the projective size of the bar and the pixel decreases with increasing photon angle, which leads to a smaller SPR value for track polar angles around $90^{\circ}$. The nonuniformity in the track azimuth angle is caused by the combinatoric background of the reconstruction procedure. The second term in Eq. 4.1 is shown on the bottom plot in Fig.4.

The obtained track resolution for the simplified design is not sufficient to meet the PANDA requirements. The dominant contribution to the SPR comes from the thickness of the bar. Therefore, either thinner bars or a focusing system are required. This method to evaluate design performance worked well, yields results consistent with expectations and will be applied to more advanced design options.
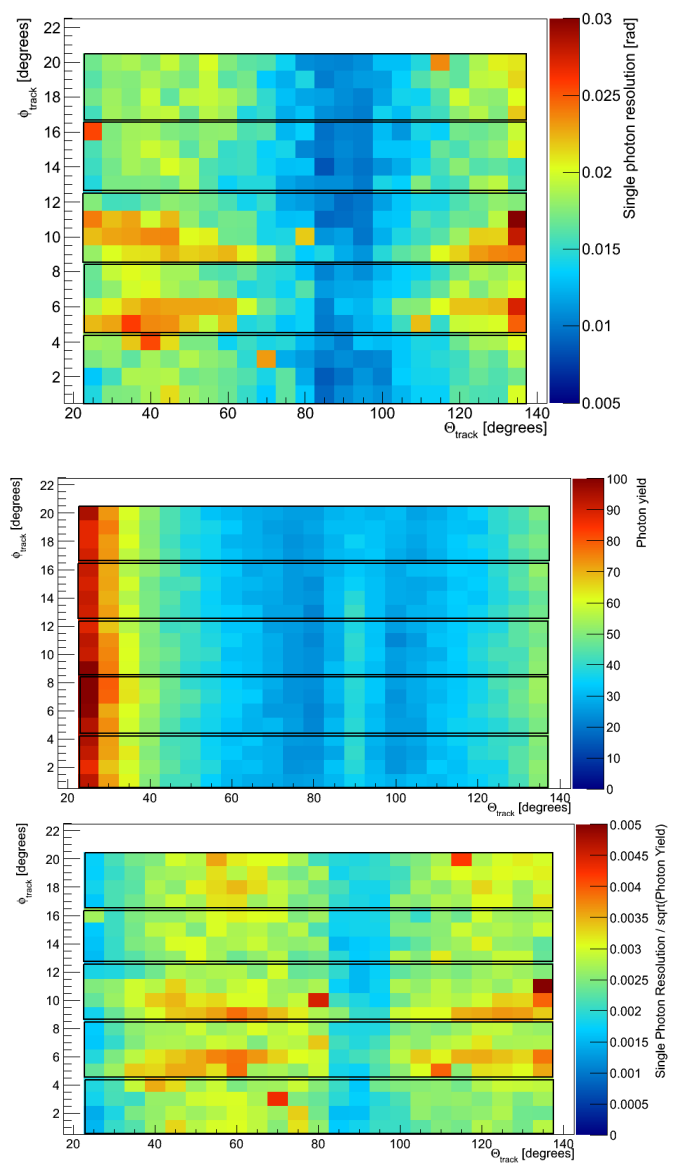

Figure 4: The maps over one bar box for the simplified design without focusing optics representing the single photon Cherenkov angle resolution (upper), the number of detected photons per track (middle) and the track resolution assuming ideal tracking (bottom). 

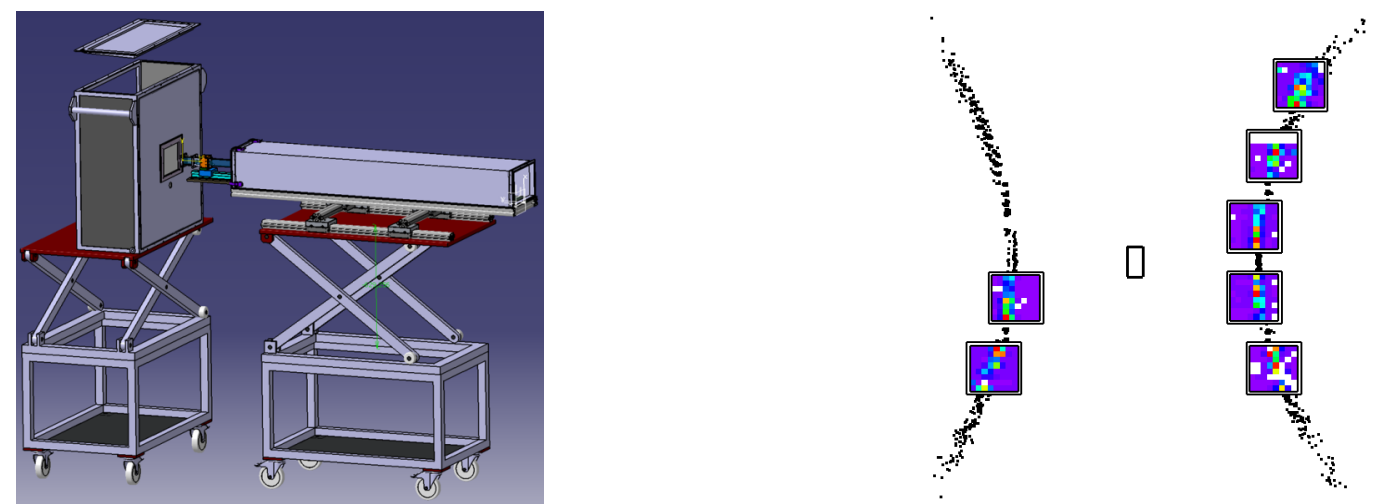

Figure 5: Prototype setup used in 2011 (on the left) and the corresponding hit pattern on the MCP-PMTs (on the right) overlaid with the simulation (black dots).

\section{Performance in particle beams}

After a design is virtually tested in the simulation, it is evaluated using particle beams. Since 2009 a number of increasingly complex Barrel DIRC prototypes have been tested in particle beams at GSI and CERN. The prototypes for the beam test campaigns 2011 and 2012 have similar components: a radiator bar, optional focusing optics, expansion volume, and photon detector array in a light-tight container. One end of the radiator bar was equipped with a front-coated mirror. The other end could be directly attached to the expansion volume using optical coupling. Alternatively, a gap between the bar and the expansion volume was introduced, where a lens could be placed. The fused silica bar together with the expansion volume were placed into a light-tight container, and an array of sensors was located on the imaging plane. The data acquisition was performed using the HADES trigger and readout board (TRB) with the TOF add-on [7], combining the NINO chip and CERN HPTDC. In order to precisely adjust the angles and the positions of the prototype, a support system with alignment stations was used. A number of lenses was tested in combination with bars produced by different companies, such as InSync Inc., Heraeus, Schott Lithotec, LZOS, and Zeiss.

In order to quantify the performance of the prototype, the SPR and the number of photons were studied for several design options and a range of track momenta and directions. The shape of the hit pattern of the prototype is defined by the geometry of the expansion volume. In the 2011 test beam campaign a large rectangular tank filled with mineral oil was used as expansion volume (Fig. 5 on the left). Photons were propagating inside it from the bar to the detector without side reflections, so the hit pattern looks like two arches of the ring. The right side of the Fig. 5 shows the observed hit pattern on the MCP-PMTs overlaid with the simulation (black dots). The reconstructed single photon Cherenkov angle resolution is in the agreement with expectation (shown in Fig. 6).

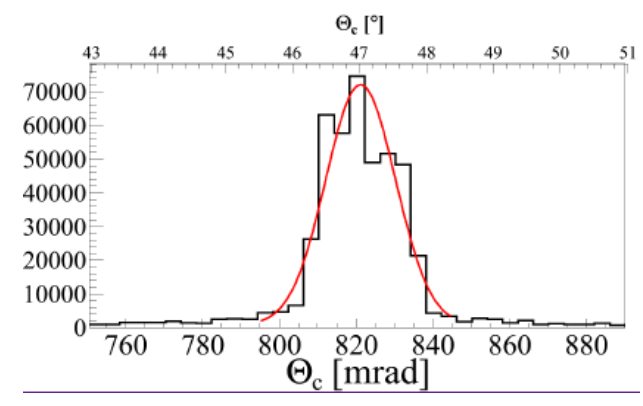

Figure 6: Reconstructed Cherenkov angle using data from 2011. The mean value and width of the Gaussian fit are $\theta_{C}=821.8 \mathrm{mrad}$ and $\sigma_{\theta_{C}}=9$ mrad, respectively, in agreement with expectation. 

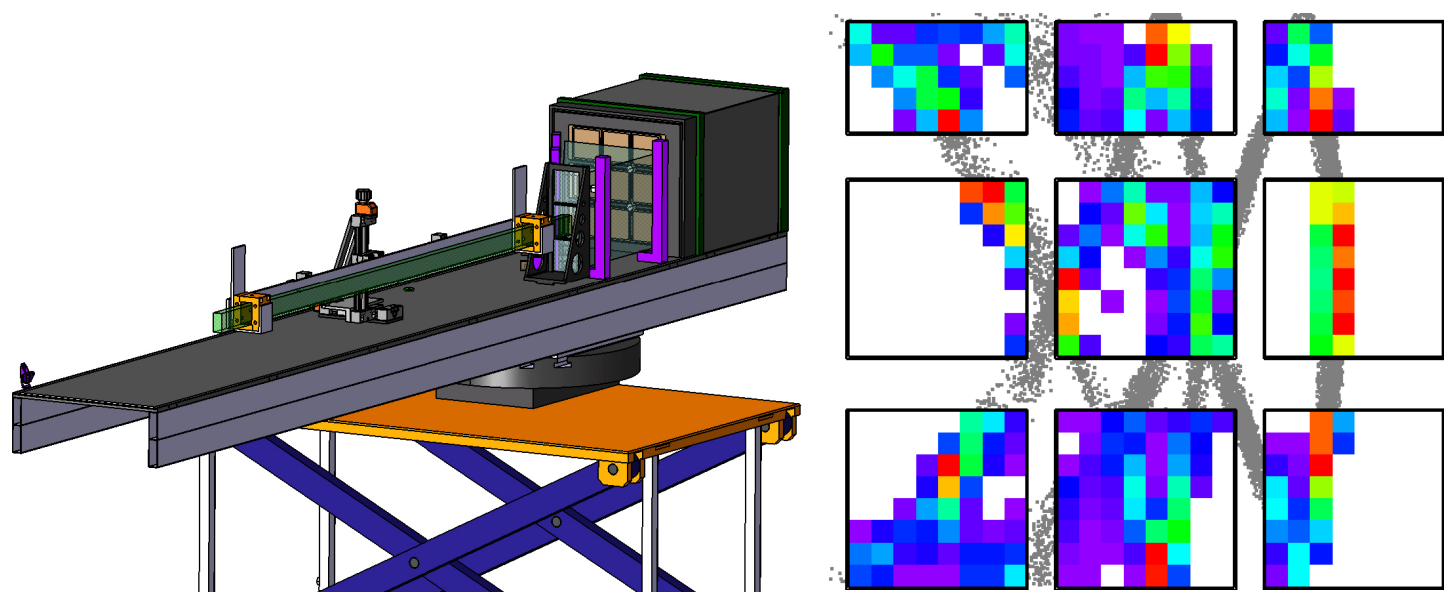

Figure 7: Prototype setup used in 2012 (on the left) and the corresponding simulated hit pattern on the MCP-PMTs (on the right). Grey points represent true photon hit locations.

In the 2012 test beam campaign a fused silica prism was used as a more compact expansion volume (see Fig. 7, on the left). Photons were reflected from the sides of the prism, resulting in more complicated hit pattern. It is consistent with the simulation (see Fig. 7, on the right). Additionally, two fiber trackers, placed before and after the radiator bar along the beam line, were used to select high quality tracks. The beam test campaign 2012 has finished just before this conference, so the data are currently being analyzed.

\section{Summary}

The hadronic PID for PANDA will be performed by two DIRC detectors, covering the barrel and forward regions. The Barrel DIRC, inspired by the successful BABAR DIRC, has several important improvements, like smaller size and more precise timing. A method to evaluate and map the performance of a detector design in terms of single photon Cherenkov angle resolution and photon yield was developed and tested on a simplified DIRC design without focusing optics. The obtained Cherenkov resolution for a track was not sufficient to satisfy the PANDA requirement. Therefore, more advanced design options including focusing systems or reduced bar thickness are currently being evaluated. A number of prototypes for the PANDA Barrel DIRC with increasing complexity have been tested in experiments using particle beams. The hit patterns and reconstructed single photon Cherenkov angle resolution observed in the 2011 data agree with simulation. The data from 2012 are currently being analyzed.

\section{References}

[1] W.F. Henning, FAIR and its experimental program, J. Phys. G 34 (2007) S551;

P. Spiller and G. Franchetti, The FAIR accelerator project at GSI.

[2] B.N. Ratcliff, The B factory detector for PEP-II: a status report, SLAC-PUB-5946 (1992); The DIRC counter: a new type of particle identification device for B factories, SLAC-PUB-6047 (1993);

P. Coyle et al., The DIRC counter: a new type of particle identification device for B factories, Nucl. Instrum. Meth. A 343 (1994) 292. 
[3] J. Schwiening et al., The Barrel DIRC detector for the PANDA experiment at FAIR, Nucl. Instrum. Meth. A 639 (2011) 315;

C. Schwarz et al., The PANDA Barrel DIRC, 2012 JINST 7 C02008.

[4] S. Agostinelli et al., Geant4 - a simulation toolkit, Nucl. Instrum. Meth. A 506 (2003) 250-303;

J. Allison et al., Geant4 developments and applications, Nuclear Science, IEEE Transactions $\mathbf{5 3}$ (2006) 270-278;

S. Spataro, Simulation and event reconstruction inside the PandaRoot framework, J. Phys. Conf. Ser. 119 (2008) 032035;

M. Al-Turany and F. Uhlig, FairRoot framework, PoS (ACAT08) 048.

[5] I. Adam et al., The DIRC particle identification system for the BABAR experiment, Nucl. Instrum. Meth. A 538 (2005) 281.

[6] M. Patsyuk, C. Schwarz, D. Dutta, K. Goetzen, K. Peters and J. Schwiening, Simulation and reconstruction of the PANDA Barrel DIRC detector (2011) JINST 6 C11015.

[7] I. Fröhlich et al., A General Purpose Trigger and Readout Board for HADES and FAIR-experiments, IEEE Trans. Nucl. Sci. 5559 (2008). 